\title{
An undergraduate collaborative team model to engage nursing students in research
}

\author{
Christine P. Kurtz ${ }^{*}$ Theresa A. Kessler \\ College of Nursing \& Health Professions, Valparaiso University, Valparaiso, Indiana, United States
}

Received: February 13, 2017

Accepted: February 21, $2017 \quad$ Online Published: February 27, 2017

DOI: $10.5430 /$ jnep.v7n7p112

URL: https://doi.org/10.5430/jnep.v7n7p112

\begin{abstract}
Purpose: Student-faculty collaboration on research is an effective model to engage undergraduate students in the research process outside the traditional curriculum. Using this model, a student-faculty team developed a longitudinal study about college students' alcohol use behaviors and implemented an intervention to influence the culture of drinking on campus.

Methods: A longitudinal design was used to assess drinking behaviors and evaluate the effect of a mass media campaign with social norm messages and alcohol education. Undergraduate students on a faith-based, Midwestern campus completed baseline $(\mathrm{N}=1,095)$ and post-intervention $(\mathrm{N}=1,011)$ electronic surveys.

Results: In addition to helping students learn about and develop enthusiasm for research, this project had an impact on the drinking culture on campus. Findings showed $88 \%$ of students observed media campaign messages with $82 \%$ viewing the printed posters, $47 \%$ viewing the outdoor displays, and $25 \%$ viewing messages on social media. There was a significant change in binge drinking from the pre- $(72 \%)$ to post-intervention (40\%) surveys.

Conclusions: Collaborative undergraduate research teams are an effective model to help students learn how to carry out research and develop interest and enthusiasm for the process. The outcomes of the project demonstrated interventions were effective at influencing the drinking culture on campus. The development of a research program outside the required curriculum can be a successful strategy to engage students in all phases of the research process, increase enthusiasm for research, and enhance health care outcomes in various settings.
\end{abstract}

Key Words: College students, Collaborative research, Alcohol use, Mass media campaign

\section{INTRODUCTION}

\subsection{Faculty-student collaborative research}

Student-faculty collaboration on research or evidence-based practice (EBP) projects is a way to help undergraduate students attain experience in and acquire a greater appreciation of these processes. ${ }^{[1]}$ Students often lack understanding of the link between research and clinical practice. Additionally, they are rarely excited about taking the required nursing research course. Fostering interest and excitement in research is a challenge for nursing educators.
The literature contains multiple examples of innovative ways of facilitating interest within the required curriculum. Educators have found that hands-on participation in research projects has greater impact than didactic classroom learning. For example, Sheriff and Chaney involved students in facultysponsored research projects as a way to facilitate interest and enthusiasm in the research process. ${ }^{[2]}$ As a graded component of the required research course, students participated in a smoking cessation study. After the experiential component was added to the course, course evaluations were very positive, and students were highly satisfied with the hands-on

*Correspondence: Christine P. Kurtz; Email: christine.kurtz@ valpo.edu; Address: College of Nursing \& Health Professions, 836 LaPorte Avenue, Valparaiso University, Valparaiso, Indiana, United States. 
participation. An added benefit was knowledge gained about the study topic, in this case smoking cessation. Through the activity, students understood how research informs clinical practice and gained appreciation and interest in the research process.

August-Brady and Adamshick used a similar strategy in their required research course, which included a weekly clinical component. ${ }^{[3]}$ Students chose clinical research projects of interest to them that were completed in the local community. Benefits of the projects included an increased respect for research, development of leadership skills, increased clinical knowledge, and development of partnerships with community agencies. Similarly, Kennel, Burns, and Horn exposed students to research by pairing them with research clinicians in clinical settings as part of the required research course. ${ }^{[4]}$ By assisting the researchers with literature reviews, data collection and analysis, and creation of poster presentations, students gained a greater understanding of research and real world patient problems. Upon evaluation, the majority of students reported a statistically significant increase in interest about nursing research.

Lesley and Chaney engaged groups of students in research by having them conduct observational studies during a clinical rotation. ${ }^{[5]}$ Students collected data while caring for patients during clinical experiences. While the methodology was not rigorous and sample sizes were small, students were able to examine relationships and find meaning in the results. Student evaluations were positive and faculty found it was an effective way for students to understand the research component of EBP.

Using qualitative methodology, Henoch and colleagues involved students in research by having them collect data for a study about patients' experience of illness. ${ }^{[6]}$ Student evaluations of the experience revealed general satisfaction with involvement in the study although some students felt unprepared for the spontaneous aspects of patient interviews. The authors noted that student involvement in only one phase of the study, data collection, may have affected the quality of learning. Involvement in all phases of the research process may be essential for deep learning to occur.

\subsection{Engaging students in research outside the curricu- lum}

The nursing literature contains fewer examples of how to engage undergraduate students in all phases of research or EBP outside the established curriculum. Experts encourage engaging students in research through independent study courses that involve faculty-led research projects. ${ }^{[1]}$ Koehler and colleagues involved students in faculty-led research through an

Published by Sciedu Press optional honors project for outstanding nursing students. ${ }^{[7]}$ Students identified topics, completed literature searches, and collected and analyzed data. Through student interviews, benefits were identified including "developing research skills, developing a professional perspective, developing a sense of achievement, and developing a passion for research" ( $p$. 6). The projects included dissemination of work via oral and poster presentations and publication of papers. One of the challenges of the projects was the high level of skill and time required of both students and faculty.

\subsection{Undergraduate collaborative team model}

At our private, faith-based university in the Midwest, nursing faculty have been leading efforts with students to plan, implement, and evaluate research projects. ${ }^{[8]}$ Faculty have a strong commitment to engaging undergraduates in the research process through collaborative projects outside the required curriculum like other science disciplines. Faculty invite students to research teams as sophomores or juniors and students are expected to participate until graduation. Senior students provide modeling and mentorship to new members. As seniors graduate, junior students move into the leadership role. Faculty act as mentors while students choose the topic for each initiative based on what they see as priorities on campus. This model provides a framework for experiential learning, immerses students in the process of knowledge generation and application of evidence, and develops leadership skills.

Recently alcohol use on campus was identified as an area of research interest. Through personal experiences as residential students and following a review of the literature, students became aware of how the campus culture normalizes and promotes alcohol use, and how this normalization may lead to dangerous consequences. Students discussed peers who were negatively affected by alcohol use, whether it was a minor consequence, such as missing class, or a more severe consequence, such as hospitalization or arrest. By working on the team and learning more about issues related to alcohol use, students became passionate about the research process and positively impacting the campus culture.

The purpose of this article is to describe an effective educational initiative used by an undergraduate student-faculty research team. The two-year collaborative project involved assessment of drinking patterns on a college campus and an intervention aimed at influencing the drinking culture.

\section{APPROACHES TO AFFECT DRINKING BE- HAVIORS}

Three main approaches to influence college students' drinking behaviors include individual strategies, environmental 
strategies, and mass media campaigns. ${ }^{[9]}$ Individual strategies generally involve one to five sessions with a healthcare provider, such as a physician, nurse, psychologist, or social worker who provides information about the risks of alcohol use, individualized feedback, social norms information, strategies for responsible drinking, discussion of positive coping skills, and goal setting. ${ }^{[10]}$ Most often, a cognitivebehavioral or motivational interviewing approach is used to help moderate alcohol use. ${ }^{[11]}$ At our university, the Office of Alcohol and Drug Education (OADE) provides brief interventions utilizing a motivational interviewing approach for individuals who have been identified as high-risk drinkers by self-referral or sanctioned legal referral.

The other two approaches, environmental strategies and mass media campaigns, are considered structural interventions. ${ }^{[9]}$ Structural interventions are used to affect change in an entire population rather than on an individual basis. Structural interventions are used within populations because there is greater potential to influence more people who may engage in risky behaviors. ${ }^{[12]}$ While students with the highest risk behaviors may require individual interventions, structural interventions have potential to prevent others from adopting risky behaviors. Environmental strategies include policies and interventions aimed at reducing alcohol use of students, both underage and those 21 years and over, and de-emphasizing the role of alcohol on campus and the surrounding community. ${ }^{[9]}$ These strategies focus on public policies and community factors as well as university policies and programs intended to impact students' drinking behaviors. ${ }^{[13]}$ The goal is to foster increased engagement in the academic aspects of college rather than in alcohol use. These strategies are provided by the OADE and the Dean's office at our university.

Mass media campaigns can be used to target behavioral norms. ${ }^{[12]}$ Mass media campaigns involve frequent messages via a combination of posters, flyers, emails, newspaper advertisements, ${ }^{[14]}$ and social media. These campaigns can focus on (a) information, i.e., increasing knowledge and awareness of problems related to alcohol use; (b) advocating for change of institutional or community policies; and (c) social norming, which involves correcting students' misperceptions about what is "normal" behavior of their peers. Social norming approaches are based on the notion that college students want to fit in with the norm of behavior on campus. ${ }^{[15]} \mathrm{Col}-$ lege students tend to overestimate peers' drinking, which may lead them to drink more to conform to what they believe is the norm. ${ }^{[15]}$ The aim of providing social norm information is to correct these misperceptions and decrease excessive drinking as a way of obtaining a sense of belonging within social groups. Results in the literature vary on the effects of this approach. In one study, social norming interventions were a successful strategy to correct perceptions of normal drinking on campuses, but the interventions did not decrease risky drinking. ${ }^{[15]}$ A meta-analysis on the use of social norm information found that while there is some significant effect, the strength of the effect was small. ${ }^{[16]}$ However, in a systematic review and meta-analysis, ${ }^{[17]}$ results demonstrated the use of social media to deliver messages had a significant positive effect on behavior change.

The first step in a media campaign is to set an objective and identify the target audience. Objectives may include influencing social and interpersonal processes. ${ }^{[14]}$ In our case, the team chose to target students' perceptions and attitudes about undergraduates' drinking behaviors. The team's objective was in alignment with the Task Force of the National Advisory Council on Alcohol Abuse and Alcoholism recommendation regarding changing the culture of drinking on college campuses. ${ }^{[18]}$ Because binge drinking is common among college students and has many dangerous consequences, the team chose to focus the campaign on this behavior. Successful media campaigns include repetition of the message and use of various media channels, especially those the target audience prefers. ${ }^{[14]}$

A mass media campaign consisting of poster distribution, postcards, and advertisements on the radio and other media was used on several college campuses to increase awareness of the risks of binge drinking. ${ }^{[19]}$ After completion of the campaign, an electronic survey and focus groups were conducted to assess awareness of the campaign and its effectiveness. Results showed that $1 / 3$ of survey respondents were aware of the campaign, and $30.4 \%$ knew the correct definition of binge drinking. Based on focus group data, students did not relate to the term "binge drinking" nor understand it as a number of drinks consumed per occasion. Rather, students believed bingeing should be defined by the intention to get drunk, speed of drinking, and tolerance for alcohol. Participants reported that images used in mass media campaigns should be realistic and relatable to the target audience and recommended use of local statistics and personal stories to create an emotional reaction. Using a variety of media was also recommended, such as television, internet, and posters.

In recent years, there has been an explosion in the use of social media, such as Twitter, Instagram, Facebook, and Snapchat. These social media platforms may be used as a tool for education and intervention to influence health behaviors such as drinking. ${ }^{[20]}$ Social networking platforms have the potential to reach large numbers of students who are at risk for dangerous alcohol consumption. How to best use these platforms has not yet been determined as research about social media is in its infancy. ${ }^{[21]}$ While social platforms such 
as Facebook and Twitter reach many people, engagement has been low. Interventions that use social media to address behavior change usually include additional methods such as flyers and advertisements. ${ }^{[21]}$

\subsection{Research team project}

Based on a review of the literature and students' awareness of a campus concern, the team decided to assess perceptions of alcohol use and binge drinking behaviors on this campus and then develop a structural intervention with the goal of impacting the drinking culture. Because individual and environmental approaches were provided within the University, the team decided to use a structural intervention with a mass media campaign and a social norm message to educate a greater number of students about alcohol consumption and increase awareness about binge drinking. Specific objectives of the team were to: (a) collect data on a sample of college students to better understand alcohol use and binge drinking behaviors, (b) analyze the relationships among variables, (c) develop structural interventions to support a change in the culture of alcohol use on campus, and (d) evaluate effects of the structural interventions.

To frame the structural intervention, the research team collected baseline data to better understand students' perceptions and use of alcohol. Data were collected using a modified version of the Core Alcohol and Drug Survey: Long

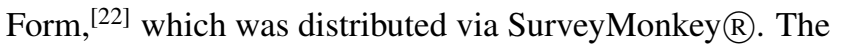
survey, which has been used nationwide, measures alcohol and other drug usage and related consequences among college students attending two and four year institutions.

The team's first structural intervention was the creation of a social norm message. Data from the baseline assessment were used to develop this messaging. The team created a poster informing students of the percentage of undergraduate students who do not use alcohol. The posters were electronic displays on televisions or laminated prints displayed throughout departmental buildings and residence halls. In addition, an advertisement was placed in the University newspaper.

During discussions with the OADE, the team learned of key events on campus associated with drinking, including Halloween weekend, Greek bid day, and spring break. The second structural intervention included a mass media campaign to coincide with each of these events. For Halloween weekend, students created outside displays of tombstones depicting statistics correlated with the consequences of binge drinking. Photos of these displays were posted on Instagram accounts of those in Greek life. Students in Greek life were targeted in particular because the baseline assessment confirmed increased rates of binge drinking among these students. For Greek bid day, the team created a laminated poster and electronic displays about the Indiana Lifeline Law (see Figure 1). For spring break, electronic and print posters defining binge drinking were displayed on campus (see Figure 2) and on Instragram accounts for student athletes and those in Greek life. The research team determined this media was used most widely by students on this campus.

Following spring break, the team created a post-intervention survey to assess the effect of the mass media campaign. It contained items from the baseline survey and also assessed students' awareness of the mass media messages and the influence of these messages.

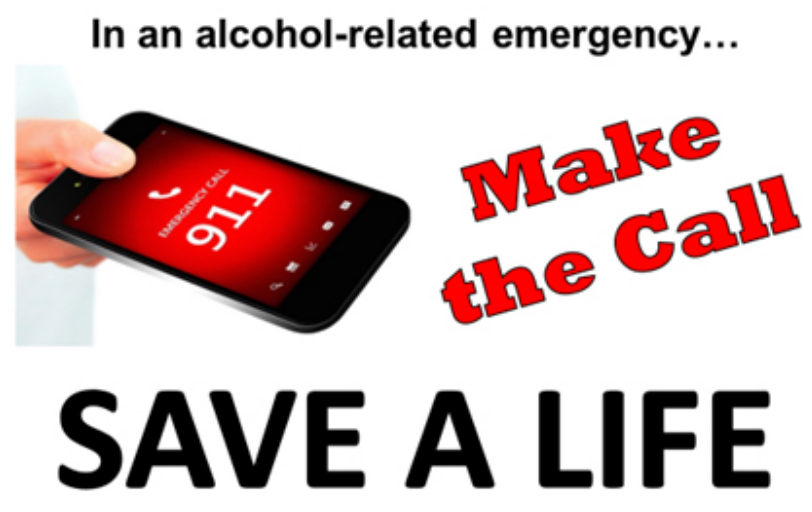

CALL. STAY. COOPERATE.

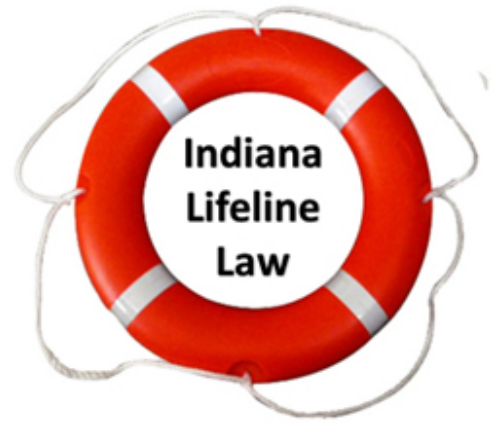

Provides IMMUNITY for public intoxication, minor possession, minor consumption, and minor transportation of alcohol, to persons who reveal themselves to law enforcement while seeking medical assistance for a person in need.

Figure 1. Indiana lifeline poster

\subsection{Analysis and dissemination of project results}

The research team's activities included analysis of data and dissemination of the research findings at conferences. Students were responsible for coding the data and entering it into SPSS, determining appropriate statistical tests to answer the research questions, and examining relationships among variables. The team created scientific posters which were 
first disseminated at the campus' celebration of undergrad- involved in research/EBP projects. The faculty's role was uate research conference. Additionally, abstracts were sent to regional and international conferences. For example, students attended the Sigma Theta Tau-International Biennial Convention as Rising Stars, a program for students who are to guide the students in dissemination activities. As a final outcome, the faculty created a manuscript for publication in a professional nursing journal.

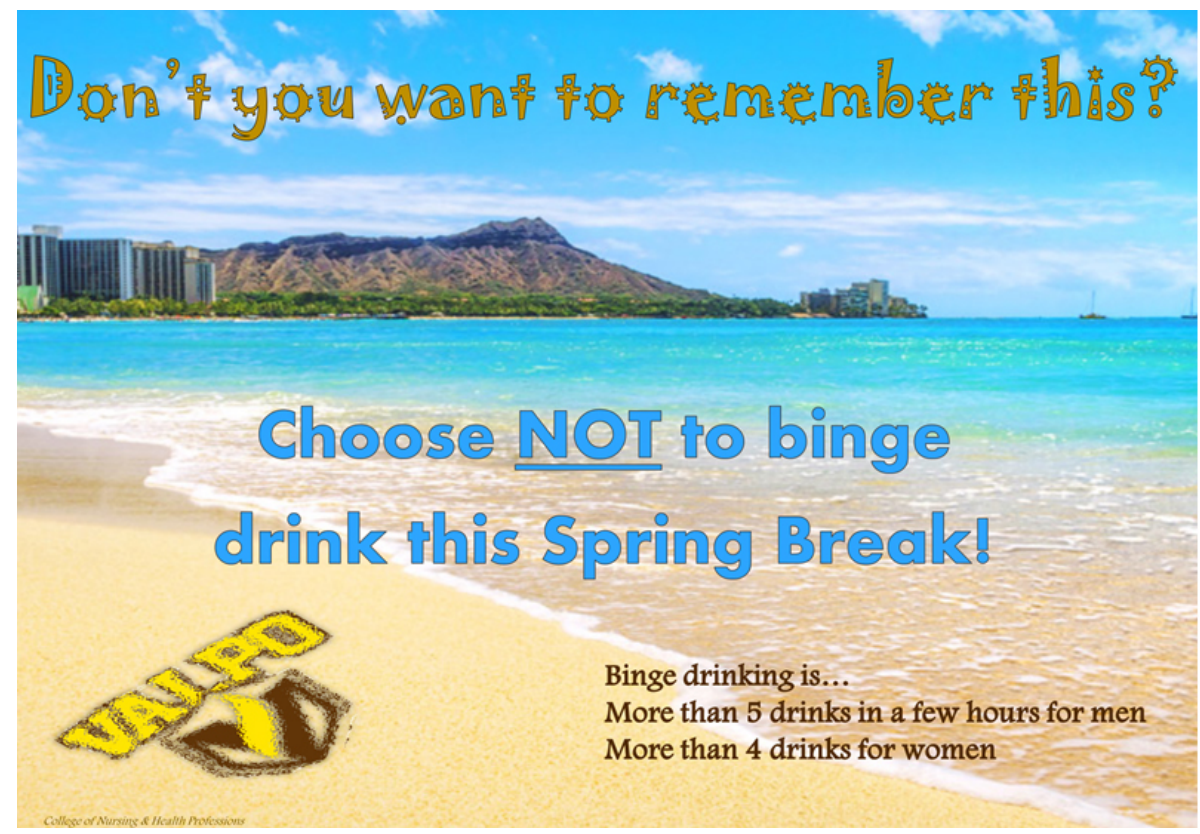

Figure 2. Definition of binge drinking poster

\section{Discussion}

Undergraduate students have the critical-thinking skills necessary to collaborate with faculty and effectively engage in research initiatives conducted outside the curriculum. This research project engaged undergraduate students in a longitudinal study to educate students about alcohol consumption and increase awareness of binge drinking on a college campus. The team chose to use a structural intervention with a mass media campaign and social norm message to meet these objectives. The student researchers managed the project through the planning, intervention, and evaluation phases. Additionally, students learned to interact with key stakeholders and various departments on campus in order to carry out the full project.

As well as learning about the research process and EBP, this project provided students the opportunity to make a positive impact on campus culture. Findings from the post-intervention assessment demonstrated the effectiveness of the intervention. A total of $88 \%$ of students in the post-intervention survey reported observing the media campaign messages with the majority (82\%) viewing the printed posters, $47 \%$ viewing the outdoor displays, and $25 \%$ viewing messages on social media. These viewing rates were much higher than thosereported in the literature in which nearly one-third of students observed the messages. ${ }^{[19]}$ Additionally, there was a significant difference in binge drinking on campus from the pre- to post-intervention survey. Of all students who drank alcohol, $72 \%$ of students reported binge drinking in the pre-intervention data compared to $40 \%$ postintervention. While causation cannot be determined by the methodology of the project, it could be that the social norm information corrected misperceptions and decreased binge drinking for some students.

Engaging students in the research process must be truly collaborative. Collaborative research projects that are not tied to required courses or to a faculty member's research agenda provide a unique opportunity for students to mature in the researcher role. This type of student learning environment fosters understanding, mutual respect, and the ability to grow and gain experience in the research. While projects led by students may not involve complex multi-factorial methodologies, undergraduate work can positively impact health outcomes. Student research projects can be conducted in local health care facilities with the potential to enhance health care outcomes in those settings. In one example from the literature, students chose to work with the homeless population by providing an evidence-based educational inter- 
vention to staff of a homeless shelter. ${ }^{[3]}$ Educating shelter staff about cues of violent behavior and appropriate intervention provided a valuable service to the homeless community. Clinicians reported that undergraduate students' involvement in clinical research was beneficial in progressing research programs in hospital settings. ${ }^{[4]}$ Similarly, Koehler and colleagues found the additional resources provided by student involvement in faculty-sponsored research, in terms of time and skills, was beneficial to advancing faculty members' research agendas. ${ }^{[6]}$ Some educators have incorporated clinical experiences into research courses allowing hands-on experience with research processes. ${ }^{[2,4]}$ In one example, pairing students with bedside clinicians who were research mentors significantly increased students' interest in research, particularly about real-world clinical problems. ${ }^{[4]}$ These students reported that being part of all phases of the project, beginning with choosing clinical questions and collecting and analyzing data, would improve the learning experience.

Involvement in the various aspects of research projects facilitates professional development. Some student projects emphasized dissemination of findings via professional conference presentations. ${ }^{[7]}$ Exposing students to these types of professional experiences as a student encourages future interest in these activities. Our research team believes it is important that students disseminate their findings at professional conferences. Attending these conferences fosters networking opportunities with peers and nurse leaders, exposes them to others' research and EBP, and provides opportunities to learn about current trends in practice. Writing manuscripts for publication has also been attempted in some programs; however, it has been found that faculty need to take the lead in this endeavor with students acting as co-authors. ${ }^{[7]}$

In addition to these benefits, collaborative projects foster enthusiasm for the researcher role. Findings in the literature demonstrate that students continue with this enthusiasm after graduation and engage in research more readily when they begin to practice nursing. ${ }^{[8]}$ One other benefit of increased enthusiasm is the potential to increase students' desire to seek graduate education. Increased numbers of nurses engaging in research and attending graduate school moves the profession forward. ${ }^{[8]}$ Students learn that research and EBP are seen as an opportunity to expand the foundation of nursing.

\section{Conclusions}

Nursing students often lack opportunities to actively participate in ongoing research as an undergraduate. An example of an effective approach is the development of collaborative student-faculty research teams. Students have the criticalthinking and skill mix to be able to take a leadership role in designing, implementing, and evaluating a program of research when mentored by faculty. ${ }^{[8]}$ At our university, student teams learn about research and develop enthusiasm for it by regularly leading all phases of research projects. An additional outcome of the projects is positively impacting health outcomes. This particular team had a positive influence on the culture of alcohol consumption and binge drinking through a structural intervention that included a mass media campaign and social norm message. The development of a research program outside the required curriculum can be a successful strategy to engage students in the research process, increase enthusiasm for research, which is often not valued by students, and enhance health care outcomes in various settings.

\section{ACKNOWLEDGeMENTS}

Undergraduate team members Jessica Mueller, Taylor Thompson, Jennifer Mitchell, Kelly Wilkins, Bradley Adkins, Ellie Ashbrook, Andrea Pertl. Research funding received from the Dr. Robert Good Grant and Kreft Endowment at Valparaiso University.

\section{CONFlicts of InTEREST Disclosure}

The authors declare that there is no conflict of interest.

\section{REFERENCES}

[1] Mission statement. [Internet]. Council on Undergraduate Research. 2016 [cited 2016 Sept 22]. Available from: http: //www . cur .org/ about_cur/

[2] Sheriff S, Chaney S. Active participation in a research study: A teaching strategy. Nurse Educ. 2006; 31(2): 53-56. PMid:16601609 https ://doi.org/10.1097/00006223-200603000-00004

[3] August-Brady M, Adamshick P. Oh, the things you will learn: Taking undergraduate research to the homeless shelter. J Nurs Educ. 2013; 52(6): 342-345.

[4] Kennel S, Burns S, Horn H. Stimulating student interest in nursing research: A program pairing students with practicing clin- ician researchers. J Nurs Educ. 2009; 48(4): 209-212. https: //doi.org/10.1097/NNE.0b013e31821611f3

[5] Lesley ML Facilitating students' success with their own research projects. Nurse Educ. 2011; 36(3): 107-111. https://doi.org/ 10.1097/NNE. 0b013e31821611f3

[6] Henoch I, Jacobsson Ung E, Ozanne A, et al. Nursing students' experiences of involvement in clinical research: An exploratory study. Nurse Educ Pract. 2014; 14: 188-194. http://dx.doi.org/10. 1016/j.nepr2013.07.009

[7] Koehler AR, Smith LR, Davies S, et al. Partners in research: developing a model for undergraduate faculty-student collaboration. Int J Nurs Scholarsh. 2015; 12(1): 1-12. 
[8] Kessler TA, Alverson EM. Mentoring undergraduate nursing students in research. Nurs Educ Perspect. 2014; 35(4): 262-64. https : //doi.org/10.5480/11-555.1

[9] Toomey TL, Lenk KM, Wagenaar A. Environmental policies to reduce college drinking: an update of research findings. J Stud Alcohol Drugs. 2007; 68: 208-219. PMid:17286339 https : //doi .org/10 $.15288 / \mathrm{j}$ sad.2007.68.208

[10] Tanner-Smith EE, Lipsey MW. Brief alcohol interventions for adolescents and young adults: a systematic review and meta-analysis. J Subst Abuse Treat. 2015; 51: 1-18.

[11] Wilke DJ, Mennicke A, Howell RL, et al. A peer-facilitated intervention to reduce risky drinking among fraternity and sorority members. J Soc Work Pract Addict. 2014; 14: 42-63. https: //doi.org/10.1080/1533256X.2014.873323

[12] Cohen DA, Scribner RA, Farley TA. A structural model of health behavior: a pragmatic approach to explain and influence health behaviors at the population level. Prev Med. 2000; 30: 146-154. PMid:10656842 https://doi.org/10.1006/pmed.1999.0609

[13] DeJong W, Langford LM. A typology for campus-based alcohol prevention: moving toward environmental management strategies. J Stud Alcohol. 2002; 14: 140-147. https ://doi.org/10.15288 /jsas.2002.s14.140

[14] DeJong W. The role of mass media campaigns in reducing high-risk drinking among college students. J Stud Alcohol. 2002; 14: 182-192. https://doi.org/10.15288/jsas.2002.s14.182

[15] Kearney B, Maley D, Mendoza R. The impact of an alcohol education program using social norming. Ky Nurse. 2013; 61(2): 6-7. PMid:23617180
[16] Foxcroft DR, Moreira MT, Santimano A, et al. Social norms information for alcohol misuse in university and college students (review) Cochrane Libr. 2015; 1.

[17] Laranjo1 L, Arguel AI, Neves AL, et al. The influence of social networking sites on health behavior change: a systematic review and meta-analysis. J Am Med Inform Assoc. 2015; 22: 243-256. PMid:25005606

[18] Drinking levels defined [Internet]. National Institute on Alcohol and Alcoholism; 2014 [cited 2014 Oct 14]. Available from: https://www.niaaa.nih.gov/alcohol-health/over view-alcohol-consumption/moderate-binge-drinking

[19] Jack SM, Sangster Bouck LM, Beynon CE, et al. Marketing a hardto-swallow message: recommendations for the design of media campaigns to increase awareness about the dangers of binge drinking. Can J Public Health. 2005; 96(3): 189-193.

[20] Scott KR, Nelson L, Meisel Z, et al. Opportunities for exploring and reducing prescription drug abuse through social media. J Addict Dis. 2015; 34: 178-184. PMid:26075802 https ://doi.org/10.1080/ 10550887.2015 .1059712

[21] Maher CA, Lewis L, Ferrar K, et al. Are health behavior changes interventions that use online social networks effective? a systematic review. J Med Internet Res. 2014; 16(2): e40. PMid:24550083 https://doi.org/10.2196/jmir. 2952

[22] Core alcohol and drug survey long form [Internet]. Southern Illinois University Carbondale/Core Institute; 2014 [cited 2014 Oct 14]. Available from: http://core.siu.edu/_common/documents/1 ongform.pdf 